Supporting Information for:

\title{
Specific Ion Effects at Calcite Surface Defects Impact
}

\section{Nanomaterial Adhesion}

Hsieh Chen, ${ }^{1, *}$ Shannon L. Eichmann, ${ }^{2}$ and Nancy A. Burnham ${ }^{3}$

${ }^{1}$ Aramco Services Company: Aramco Research Center-Boston, Cambridge, MA, 02139, U.S.A.

${ }^{2}$ Aramco Services Company: Aramco Research Center-Houston, Houston, TX, 77084, U.S.A.

${ }^{3}$ Physics and Biomedical Engineering Departments, Worcester Polytechnic Institute, Worcester, MA, 01609, U.S.A.

"hsieh.chen@aramcoamericas.com 


\section{S1. Supplementary Results and Discussion}

\section{S1.1. Hydration Structures near Surface Defects}

Fig. S1a shows the radial distribution function $g(r)$ between water molecules and the calcite steps. Interestingly, we observed that the tightly bound first and second hydration layers were located at similar distances on both steps (near $0.3 \mathrm{~nm}$ ), but the third to sixth hydration layers were all located slightly farther away from the obtuse step compared to the acute step (c.f. stars noted in Fig. S1a). To the best of our knowledge, this is the first study showing changes in long-range differential water structures up to the sixth water layer at $r \sim 1.5 \mathrm{~nm}$ induced by the line defects on calcite surfaces. More research may be necessary in this area to elucidate the implications for these water structures beyond their differential affinity for nanomaterial adhesion.

Fig. S1b shows the hydration structures near the vacancies. The vacancies perturb the water structures until the third hydration layer $(r \sim 0.6 \mathrm{~nm})$, which was very different from the water structures perturbed by the line defects (Fig. S1a). The observation that line defects lead to more order in the hydration structure than the point defects may be an area for further study. 


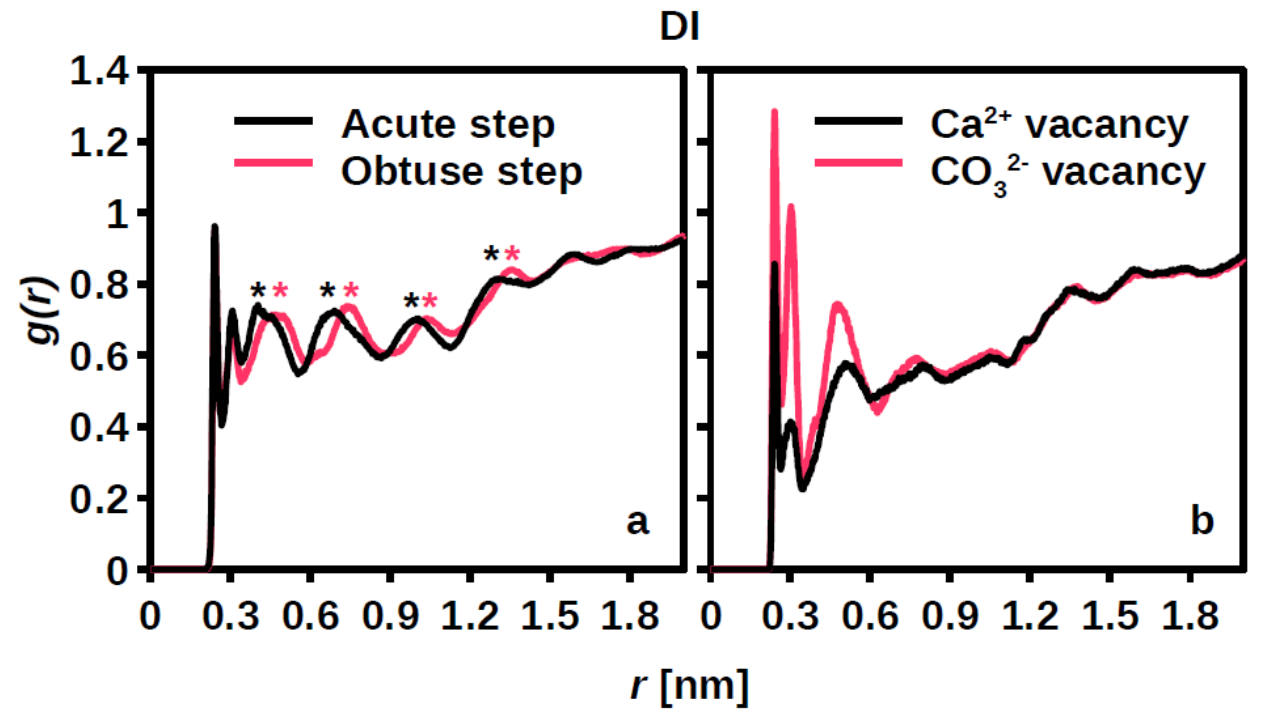

Fig. S1. Radial distribution functions $g(r)$ for the water molecules near (a) line and (b) point defects in DI. The third to the sixth hydration layer are noted with stars in (a) showing the long-range differential hydration structures on the line defects. 


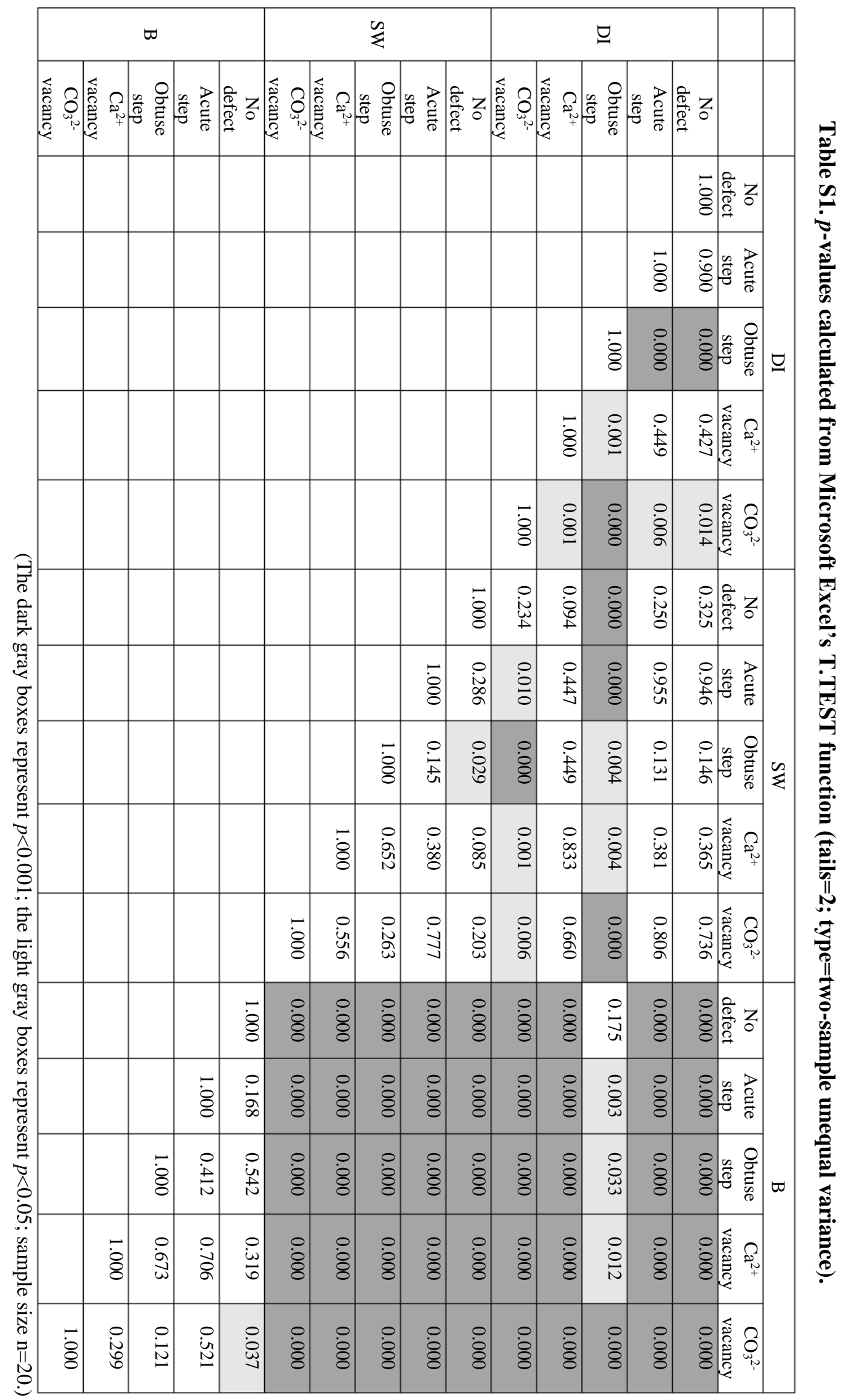

